\title{
STRAINS AND TILTS ON THE SURFACE OF A SEMI-INFINITE MEDIUM
}

\author{
Ryosuke Sato and Mitsuhiro MATSU'URA \\ Geophysical Institute, Faculty of Science, \\ University of Tokyo, Tokyo, Japan
}

(Received April 18, 1974)

\begin{abstract}
Analytical expressions for the strain and tilt fields due to an inclined rectangular fault in a semi-infinite medium with an arbitrary Poisson's ratio are derived. Since only simple algebraic functions are involved, numerical computation can be quickly carried out.
\end{abstract}

\section{Introduction}

The source mechanisms have been successfully discussed by comparing not only dynamic measurements such as seismic body waves, surface waves and tsunami waves but also static measurements such as residual displacement and strain fields, with corresponding theoretical values.

Chinnery (1961, 1963) and Maruyama (1964) used Steketee's (1958) results in calculating the near static displacement field for a vertical rectangular strike-slip and dip-slip fault. Expressions for the residual strain and tilt fields are given by Press (1965) for a vertical fault. MANsinha and Smylie (1971) derived analytical expressions of the displacement field due to an inclined fault, assuming Poisson's ratio of 0.25 . These expressions are conveniently used by many investigators in the interpretation of observed static fields associated with earthquakes.

Recently strainmeters and tiltmeters have been installed in many places mainly from necessity of research in earthquake prediction. To interprete these "zero frequency measurements" in detail comparing with theoretical values, it is necessary to calculate them for a realistic structure. Even if they could be found in a realistic structure, say, a multi-layered medium, it would take rather a long computational time even for calculating one component. In many cases, however, an assumption of a semi-infinite medium is sufficient in the interpretation of observations available at least at present. If theoretical values in a semi-infinite medium can be quickly calculated without using numerical double integration technique, they can also be used as diagnostic of those in a multi-layered medium.

In the present paper, closed analytical expressions for the strain and tilt 
fields due to an inclined rectangular fault in a semi-infinite medium with an arbitrary Poisson's ratio are obtained by which the fields are quite easily computed and contoured. AlEWINE (personal communication, 1974) independently derived the strain fields by differentiating expressions given by Mansinha and Smylie (1971) for a Poisson solid.

\section{Strain and Tilt Fields}

Take the Cartesian coordinate system $(x, y, z)$ where the half space occupies the region $z<0$. When a point fault is located at $(0,0,-d)$, static displacements at the surface $(z=0)$ are expressed, after some modifications of SATo's (1971) results, by

$$
\left.\begin{array}{l}
u_{x}^{0}=-\frac{U_{0}}{2 \pi}\left[\sin \lambda\left\{\frac{3 x p q}{R^{5}}-\frac{\partial F_{1}}{\partial x} \sin \delta \cos \delta\right\}+\cos \lambda\left\{\frac{3 x^{2} q}{R^{5}}+\frac{\partial F_{2}}{\partial x} \sin \delta\right\}\right], \\
u_{y}^{0}=-\frac{U_{0}}{2 \pi}\left[\sin \lambda\left\{\frac{3 y p q}{R^{5}}-\frac{\partial F_{1}}{\partial y} \sin \delta \cos \delta\right\}+\cos \lambda\left\{\frac{3 x y q}{R^{5}}+\frac{\partial F_{2}}{\partial y} \sin \delta\right\}\right], \\
u_{z}^{0}=-\frac{U_{0}}{2 \pi}\left[\sin \lambda\left\{\frac{3 d p q}{R^{5}}-\frac{\partial F_{3}}{\partial x} \sin \delta \cos \delta\right\}+\cos \lambda\left\{\frac{3 x d q}{R^{5}}+\frac{\partial F_{3}}{\partial y} \sin \delta\right\}\right],
\end{array}\right\}
$$

where

$$
\begin{aligned}
& \left.\begin{array}{l}
p=y \cos \delta+d \sin \delta, \\
q=y \sin \delta-d \cos \delta,
\end{array}\right\} \\
& R=\left(r^{2}+d^{2}\right)^{1 / 2}=\left(x^{2}+y^{2}+d^{2}\right)^{1 / 2}, \\
& F_{1}=\frac{1-\alpha}{\alpha}\left\{\frac{x^{2}-y^{2}}{r^{4}}(R-d)-\frac{x^{2}}{r^{2} R}\right\}, \\
& F_{2}=\frac{1-\alpha}{\alpha}\left\{\frac{2 x y}{r^{4}}(R-d)-\frac{x y}{r^{2} R}\right\}, \\
& F_{3}=\frac{1-\alpha}{\alpha} \frac{x}{r^{2}}\left(1-\frac{d}{R}\right), \\
& \alpha=1-\left(V_{\mathrm{S}} / V_{\mathrm{P}}\right)^{2},
\end{aligned}
$$

$U_{0}$ is the amount of displacement discontinuity, $\delta$ the dip-angle, $\lambda$ the angle between $x$-axis and slip direction, and $V_{\mathrm{P}}$ and $V_{\mathrm{S}}$ are $\mathrm{P}$ and $\mathrm{S}$ velocities.

For a fault with length $2 L$ and width $W$ (Fig. 1), the displacement fields can be found by taking $x-\xi, y-\eta \cos \delta$ and $d-\eta \sin \delta$ in place of $x, y$ and $d$ in (1) and then applying an integration operator

$$
\int_{-L}^{L} \mathrm{~d} \xi \int_{0}^{W} \mathrm{~d} \eta
$$




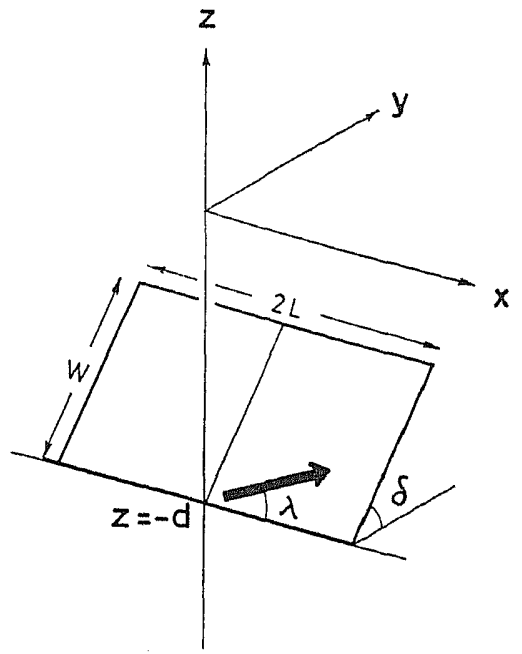

Fig. 1. Fault geometry and coordinate system.

Changing variables from $\xi, \eta$ to $\xi^{\prime}, \eta^{\prime}$ by

$$
\left.\begin{array}{l}
x-\xi=\xi^{\prime}, \\
p-\eta=\eta^{\prime},
\end{array}\right\}
$$

the above integration operator becomes

$$
\left.\begin{array}{c}
\int_{x-L}^{x+L} \mathrm{~d} \xi^{\prime} \int_{p-W}^{p} \mathrm{~d} \eta^{\prime}, \\
y-\eta \cos \delta=\eta^{\prime} \cos \delta+q \sin \delta, \\
d-\eta \sin \delta=\eta^{\prime} \sin \delta-q \cos \delta,
\end{array}\right\}
$$

The integration of derivatives of displacements with respect to $x$ or $y$ can be done without difficulty and the results, expressed in indefinite integral forms, are given in the following:

The strains are

$$
\begin{aligned}
\frac{\partial u_{x}}{\partial x}= & \frac{U_{0}}{2 \pi} \sin \lambda\left[\frac{\xi^{\prime} q}{R^{3}}+I_{1} \sin \delta \cos \delta\right]+\frac{U_{0}}{2 \pi} \cos \lambda\left[\xi^{\prime 2} q A_{\eta^{\prime}}-I_{2} \sin \delta\right], \\
\frac{\partial u_{x}}{\partial y}= & -\frac{U_{0}}{2 \pi} \sin \lambda\left[\frac{1}{R^{3}}\left\{\xi^{\prime 2} \sin \delta+\eta^{\prime} D\right\}-I_{2} \sin \delta \cos \delta\right] \\
& +\frac{U_{0}}{2 \pi} \cos \lambda\left[\xi^{\prime 3}\left\{\frac{D}{Y^{2} R^{3}}-A_{\eta^{\prime}} \sin \delta\right\}\right. \\
& \left.+\frac{1-\alpha}{\alpha} \frac{\xi^{\prime}}{R\left(R+\eta^{\prime}\right)} \sin \delta+I_{1} \sin \delta\right],
\end{aligned}
$$




$$
\begin{aligned}
& \frac{\partial u_{u}}{\partial x}=\frac{U_{0}}{2 \pi} \sin \lambda\left[\frac{q}{R^{3}}\left\{q \sin \delta-\frac{\eta^{\prime 3}}{X^{2}} \cos \delta\right\}+I_{2} \sin \delta \cos \delta\right] \\
& +\frac{U_{0}}{2 \pi} \cos \lambda\left[\xi^{\prime} q\left\{q A_{\eta^{\prime}} \sin \delta+\frac{1}{R^{3}} \cos \delta\right\}\right. \\
& \left.+\frac{1-\alpha}{\alpha} \frac{\xi^{\prime}}{R\left(R+\eta^{\prime}\right)} \sin \delta+I_{1} \sin \delta\right] \text {, } \\
& \frac{\partial u_{y}}{\partial y}=-\frac{U_{0}}{2 \pi} \sin \lambda\left[\frac{\xi^{\prime} q}{2 R^{3}}+\left\{\frac{1}{2 R}\left(\frac{\xi^{\prime}}{R+\eta^{\prime}}+\frac{2 \eta^{\prime}}{R+\xi^{\prime}}\right)-\eta^{\prime} q^{2} A_{\xi^{\prime}}\right\} \sin 2 \delta\right. \\
& -q\left\{\frac{1}{R\left(R+\xi^{\prime}\right)}+\frac{1}{2}\left(\eta^{\prime 2}-q^{2}\right) A_{\xi^{\prime}}\right\} \cos 2 \delta \\
& \left.+\frac{1-\alpha}{\alpha} \frac{\xi^{\prime}}{R\left(R+\eta^{\prime}\right)} \sin \delta \cos \delta+I_{1} \sin \delta \cos \delta\right] \\
& -\frac{U_{0}}{2 \pi} \cos \lambda\left[\frac{1}{2} \xi^{\prime 2} q A_{\eta^{\prime}}+\frac{\xi^{\prime 2}+\eta^{\prime 2}-q^{2}}{2 R^{3}} \sin 2 \delta\right. \\
& -q\left\{\frac{\eta^{\prime}}{2 R^{3}}+\frac{1}{R\left(R+\eta^{\prime}\right)}-\frac{1}{2} q^{2} A_{\eta^{\prime}}\right\} \cos 2 \delta \\
& \left.-\frac{1-\alpha}{\alpha} \frac{1}{R}\left\{\frac{q}{R+\eta^{\prime}} \sin \delta+\cos \delta\right\} \sin \delta-I_{2} \sin \delta\right] \text {, }
\end{aligned}
$$

where

$$
\left.\begin{array}{c}
I_{1}=\int \frac{\partial F_{1}}{\partial x} \mathrm{~d} \eta^{\prime}=\frac{1-\alpha}{\alpha} \frac{\xi^{\prime}}{X^{2} R(R+D)^{2}}\left[\eta^{\prime} Y^{2}+R\left\{\eta^{\prime} D-q Y_{1}\right\}\right], \\
I_{2}=\int \frac{\partial F_{1}}{\partial y} \mathrm{~d} \eta^{\prime}=-\frac{1-\alpha}{\alpha} \frac{1}{2 X^{2} R(R+D)^{2}}\left[\xi^{\prime 2}\left\{2 \eta^{\prime} Y_{1}-q(R-D)\right\}\right. \\
\left.+q(R+D)\left(R^{2}+Y^{2}\right)\right]
\end{array}\right\}
$$

The tilts are

$$
\begin{aligned}
\frac{\partial u_{z}}{\partial x}= & -\frac{U_{0}}{2 \pi} \sin \lambda\left[\frac{q}{R^{3}}\left\{\frac{\eta^{3}}{X^{2}} \sin \delta+q \cos \delta\right\}-I_{3} \sin \delta \cos \delta\right] \\
& +\frac{U_{0}}{2 \pi} \cos \lambda\left[\frac{\xi^{\prime} q}{R^{3}}\left\{\sin \delta+\frac{\eta^{\prime} q\left(3 X^{2}+2 \eta^{\prime 2}\right)}{X^{4}} \cos \delta\right\}-I_{4} \sin \delta\right],
\end{aligned}
$$




$$
\begin{aligned}
\frac{\partial u_{z}}{\partial y}=-\frac{U_{0}}{2 \pi} & \sin \lambda\left[\frac{1}{2 R}\left\{\frac{\xi^{\prime}}{R+\eta^{\prime}}+\frac{2 \eta^{\prime}}{R+\xi^{\prime}}\right\}-q\left\{\frac{\xi^{\prime}}{2 R^{3}}+\eta^{\prime 2} A_{\xi^{\prime}}\right\} \sin 2 \delta\right. \\
& \left.-\frac{1}{2}\left\{\frac{\xi^{\prime} \eta^{\prime}}{R^{3}}+\frac{\xi^{\prime}}{R\left(R+\eta^{\prime}\right)}+\eta^{\prime}\left(\eta^{\prime 2}-q^{2}\right) A_{\xi^{\prime}}\right\} \cos 2 \delta-I_{4} \sin \delta \cos \delta\right] \\
& -\frac{U_{0}}{2 \pi} \cos \lambda\left[\frac{1}{2 R}-q\left\{\frac{\eta^{\prime}}{R^{3}}+\frac{1}{2} \xi^{\prime 2} A_{\eta^{\prime}}\right\} \sin 2 \delta-\frac{\xi^{\prime 2}+\eta^{\prime 2}-q^{2}}{2 R^{3}} \cos 2 \delta\right. \\
& \left.-\frac{1-\alpha}{\alpha} \frac{1}{R}\left\{\sin \delta-\frac{q}{R+\eta^{\prime}} \cos \delta\right\} \sin \delta-I_{3} \sin \delta\right]
\end{aligned}
$$

where

$$
\left.\begin{array}{l}
I_{3}=\int \frac{\partial F_{3}}{\partial x} \mathrm{~d} \eta^{\prime}=-\frac{1-\alpha}{\alpha} \frac{1}{X^{2} R(R+D)}\left[\xi^{\prime 2} \eta^{\prime}+R q Y_{1}\right], \\
I_{4}=\int \frac{\partial F_{3}}{\partial y} \mathrm{~d} \eta^{\prime}=-\frac{1-\alpha}{\alpha} \frac{\xi^{\prime}}{X^{2} R(R+D)}\left[\eta^{\prime} Y_{1}-R q\right] .
\end{array}\right\}
$$

\section{Numerical Example}

Discussion of general behabiors of the strain and tilt fields for various fault models is not the purpose of the present paper. Here one numerical example is shown.

KANAMORI (1974) proposed "hypothetical earthquake" in the vicinity of Kanto District, Japan (Fig. 2), taking account of period of major earthquake occurrence and other evidences in this area and computed ground displacements at Tokyo. The focal parameters for this hypothetical earthquake are

$$
\lambda=\tan ^{-1}(-0.65 /+2.0)=-18^{\circ}
$$

(right-lateral strike slip of $2 \mathrm{~m}$ and reverse dip slip of $0.65 \mathrm{~m}$ ),

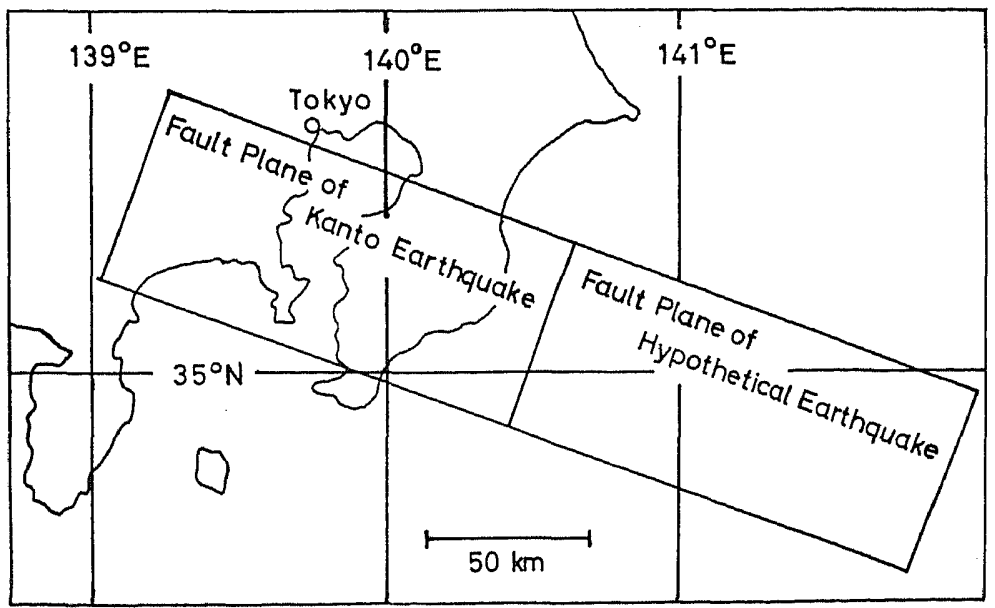

Fig. 2. Fault planes of the 1923 Kato Earthquake and a hypothetical earthquake (after KANAMORI, 1974) 


$$
\begin{aligned}
\delta & =-34^{\circ}, \\
L & =65 \mathrm{~km}, \\
W & =70 \mathrm{~km}, \\
U_{0} & =2.1 \mathrm{~m} .
\end{aligned}
$$

In the present calculation, the focal depth $d$ (Fig. 1) is taken as $0.1 \mathrm{~km}$ and $V_{\mathrm{P}} / V_{\mathrm{S}}$ as $\sqrt{3}$. Figure 3 illustrates the strain and tilt fields due to the hypothetical earthquake. Numerals on contour curves are shown in unit of $2.1 \times$ $10^{-5}$. High strains and tilts are seen in the eastern part of Boso Peninsula.

The machine program is available to compute and contour the strain and tilt fields as well as the displacement field on the surface of a semi-infinite medium and is open to the use by anybody.

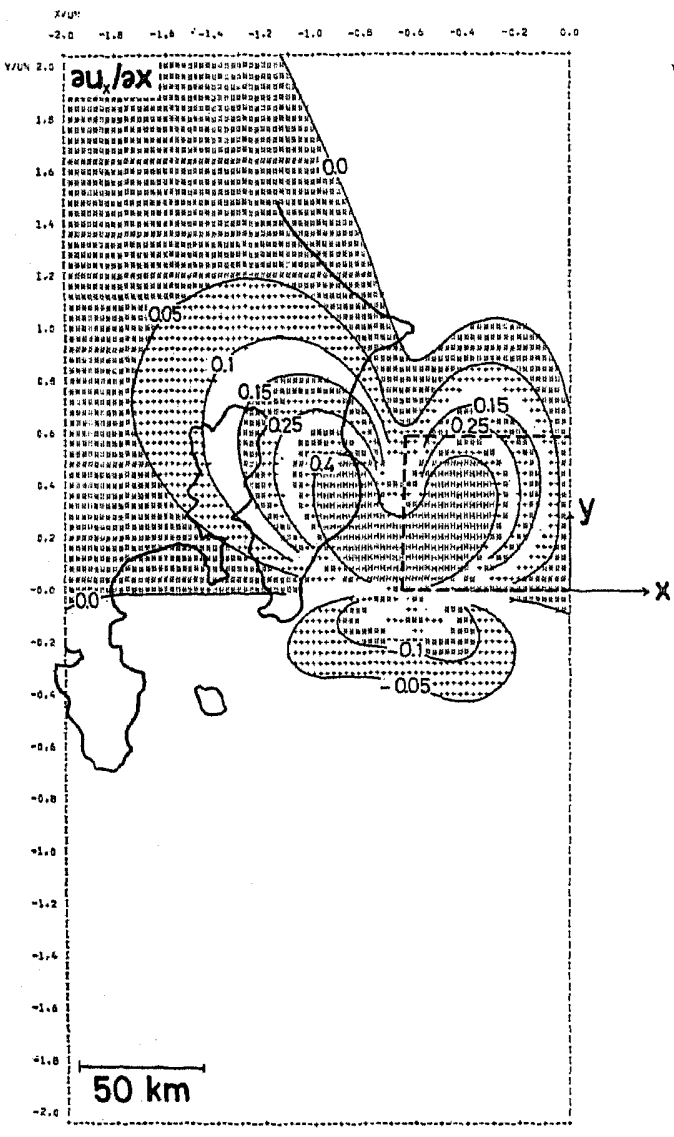

(a)

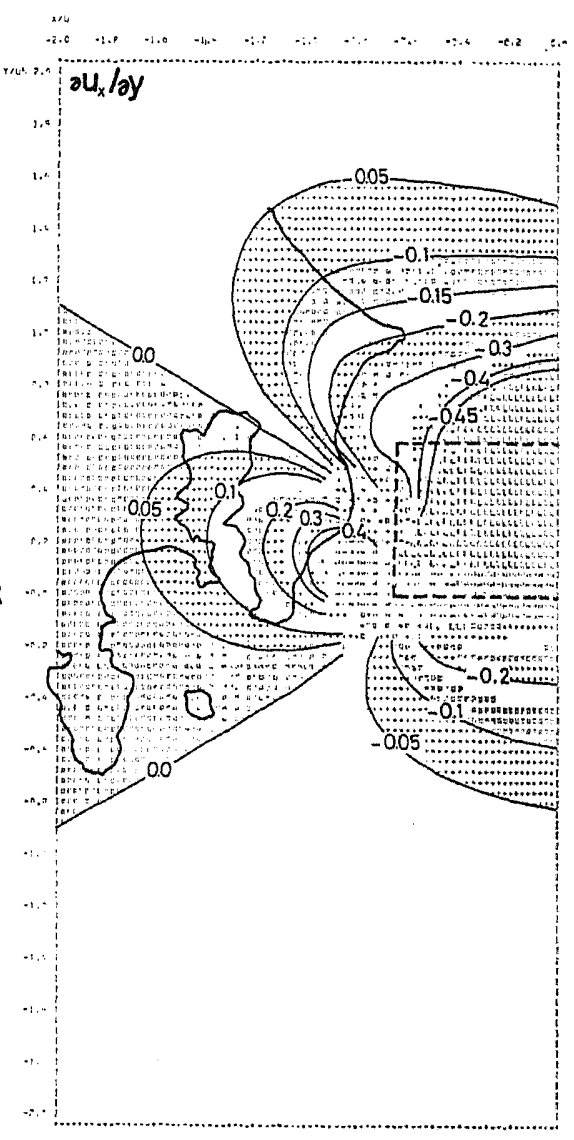

(b)

Fig. 3. 


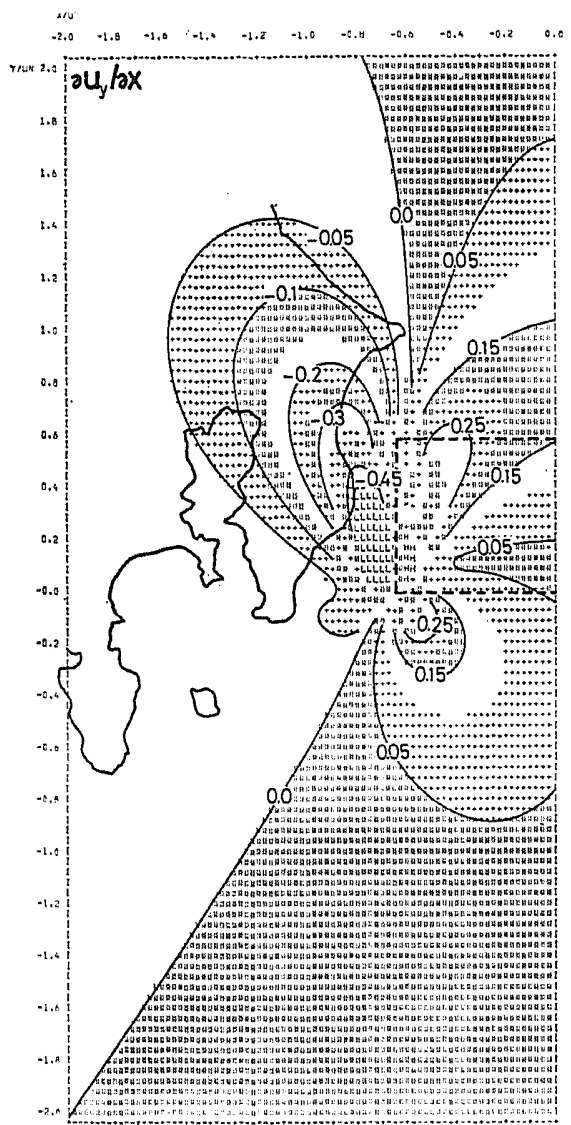

(c)

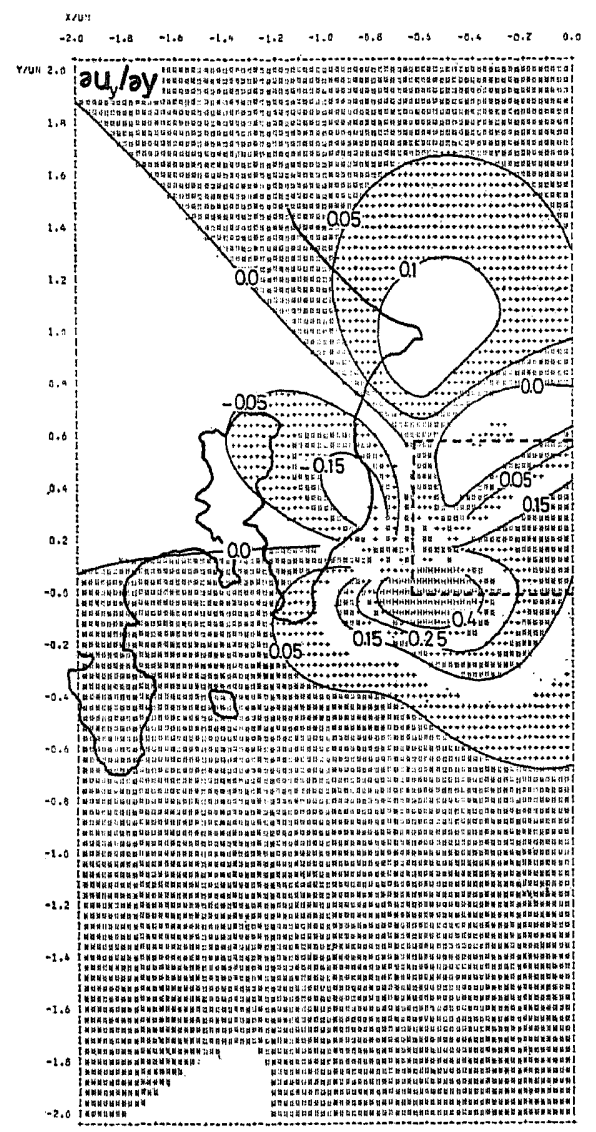

(d)

Fig. 3. The strain and tilt fields due to the hypothetical earthquake proposed by Kanamori. 


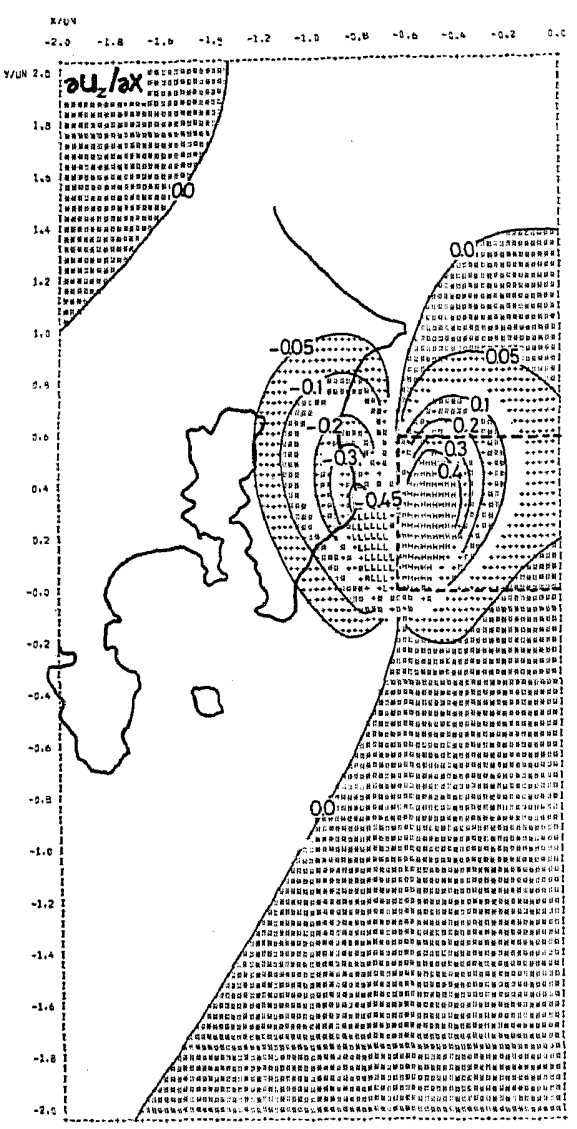

(e)

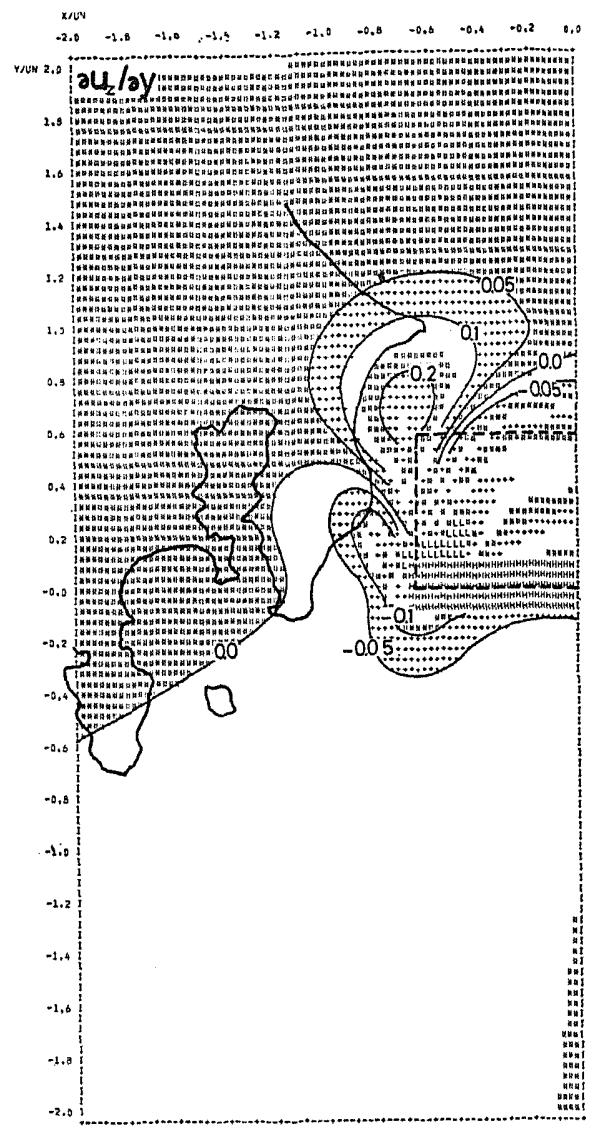

(f)

Fig. 3.

\section{REFERENCES}

AlEwine, R. W., Application of linear inversion theory toward the estimation of seismic source parameters, Ph. D. Thesis, California Institute of Technology, Pasadena, 303 pp., 1974.

Chinnery, M. A., The deformation of the ground around surface faults, Bull. Seism. Soc. Am., 51, 355-372, 1961.

Chinnery, M. A., The stress changes that accompany strike-slip faulting, Bull. Seism. Soc. Am., 53, 921-932, 1963.

KANAMORI, H., Long-period ground motion in the epicentral area of major earthquakes, Tectonophysics, 21, 341-356, 1974.

Mansinha, L. and D. E. Smylie, The displacement fields of inclined faults, Bull. Seism. Soc. Am., 61, 1433-1440, 1971.

Maruyama, T., Statical elastic dislocations in an infinite and semi-infinite medium., Bull. Earthq. Res. Inst., 42, 289-368, 1964. 
Press, F., Displacements, strains, and tilts at teleseismic distances, J. Geophys. Res., 70, 23952412, 1965.

SAto, R., Crustal deformation due to dislocation in a multi-layered medium, $J$. Phys. Earth, 19, 31-46, 1971.

Steketee, J. A., On Volterra's dislocations in a semi-infinite medium, Can. J. Phys, , 36, 192$205,1958$. 